\title{
Definition and Classification of Intraoperative Complications (CLASSIC): Delphi Study and Pilot Evaluation
}

\author{
Rachel Rosenthal · Henry Hoffmann • \\ Pierre-Alain Clavien · Heiner C. Bucher • \\ Salome Dell-Kuster
}

Published online: 10 February 2015

(C) Société Internationale de Chirurgie 2015

\begin{abstract}
Background Standardized reporting of intraoperative adverse events is important to enhance transparency. To the best of our knowledge, there is no validated definition and classification of intraoperative complications.

Methods We conducted a two-round Delphi study to develop a definition and classification of intraoperative complications. Experts were contacted by email and sent a link to the online questionnaire. In a pilot study, two independent raters applied the definition and classification in a sample of 60 surgical interventions of low, intermediate, and high complexity and evaluated practicability. Interrater agreement of the classification was determined (raw categorical agreement, weighted kappa, and intraclass correlation).

Results In the Delphi study, 40 of 52 experts (77\% return rate) from 14 countries took part in each round. The Delphi study resulted in a comprehensive definition of intraoperative complications as any deviation from the ideal intraoperative course occurring between skin incision and skin closure. The classification foresees four grades depending on the need for treatment (no need, grade I; need for treatment, grade II) and the severity of the complication (life-threatening/permanent disability, grade III; death, grade IV). The pilot study showed good practicability (6 on a 7-point scale) and a high raw agreement of $87 \%$, a weighted kappa of 0.83 [95\% confidence interval (CI) 0.73-0.94] and an intraclass correlation coefficient of 0.83 (95\% CI 0.73-0.90).

Conclusions While the Delphi process enabled to develop definitions and classification of intraoperative complications by severity, further research including a multicentre international full-scale validation needs to be conducted with the ultimate goal to contribute to standardized reporting in surgical practice and research.
\end{abstract}

Electronic supplementary material The online version of this article (doi:10.1007/s00268-015-3003-y) contains supplementary material, which is available to authorized users.

R. Rosenthal $(\bowtie) \cdot$ H. Hoffmann · S. Dell-Kuster

Department of Surgery, University Hospital Basel, 4031 Basel,

Switzerland

e-mail: rachel.rosenthal@unibas.ch

\section{P.-A. Clavien}

Department of Surgery, University Hospital Zurich and

University of Zurich, Zurich, Switzerland

\section{Introduction}

Reporting of surgical outcomes is important for informed decision making both in clinical research and in clinical practice [1-5]. This not only includes efficacy parameters,

H. C. Bucher · S. Dell-Kuster

Basel Institute for Clinical Epidemiology and Biostatistics,

University Hospital Basel, Basel, Switzerland

S. Dell-Kuster

Department of Anesthesiology, University Hospital Basel, Basel, Switzerland 
but also adverse events, i.e., intra- and postoperative complications. For postoperative complications, several definitions and classification systems have been proposed [6-11], whereas we are not aware of any validated reporting system addressing intraoperative complications.

A critical appraisal of the current practice of reporting intra- and postoperative complications in a cohort of 46 randomized controlled trials published in 2010 in the Annals of Surgery, JAMA Surgery, and the BJS revealed that $41 \%$ of the trials failed to report intraoperative complications [12]. Only $13 \%$ provided a definition and $9 \%$ used a classification for intraoperative complications.

The aim of the present study was to develop a definition and classification for intraoperative complications within a Delphi study and to conduct a pilot study evaluating practicability and interrater agreement.

\section{Materials and methods}

Delphi study

\section{Participants}

International experts with a surgical or methodological background were invited to participate in the Delphi study with the intention to develop a classification applicable to any surgical subspecialty. Experts were recruited through personal contacts and surgical associations with the goal to get a representative sample of different expertise and location of practice across the world.

\section{Questionnaire dissemination}

This Delphi study consisted of two rounds (February and March 2014). The questionnaires were prepared using SurveyMonkey ${ }^{\circledR}$ (www.surveymonkey.com). The experts were sent a link to the online questionnaire by email. The anonymous responses were downloaded as Excel spreadsheet (Microsoft Office XP, Microsoft Corporation, Redmond, WA, USA). One email reminder per round was sent out before the end of the deadline to all experts, since the anonymous process did not allow tracking responders.

\section{Content first round}

Since there is abundant literature on the classification of postoperative complications, we refrained from a first round with open question [13].

Participants were provided with some background information about surgical complications and were asked to fill in their baseline demographics. They were then invited to indicate their level of agreement on a 9-point rating scale with the inclusion of a number of items concerning the definition and classification of intraoperative complications. We chose a 9-point scale because scales with too low numbers of items, such as two- to four-point scales, have been found to show poor reliability, validity, and discriminating power [14]. Conversely, the known limit in accuracy of raters to give an absolute judgement is at about seven categories [15]; we rounded up to nine in order to account for a potential end-aversion bias (i.e., have two extra categories to address the tendency to avoid extreme categories). Raters could not move on with the questionnaire without having rated every item. Additionally, experts were strongly encouraged to comment on any of the items.

\section{Content second round}

The quantitative and qualitative results of the first round were presented to the participants and where applicable the questions were updated accordingly. Since sufficient consensus was obtained after two rounds, no third round was conducted.

\section{Data analysis}

Descriptive statistics including graphs were used to analyze quantitative data. All statistical analyses were conducted using Intercooled Stata Version 12.1; StataCorp LP, College Station, Texas, USA. Comments (qualitative data) were summarized within a narrative review. Since the questionnaire prompted extensive comments and a fruitful discussion, the manuscript only contains the main findings.

Pilot study

\section{Surgical interventions}

The pilot study was conducted in a retrospective cohort of patients operated in 2013 in a tertiary referral teaching hospital. A random sample of 60 records of patients having undergone surgeries of different complexity (20 type A, 20 type B, and 20 type C) was selected. Type A, B, and C surgery was defined according to previous investigations $[8,16]$. In brief, type A surgery includes surgical procedures without opening the abdominal cavity. Type B surgery includes abdominal procedures with the exception of liver surgery and major retroperitoneal surgery. Type C surgeries include highly specialized procedures such as surgery on the liver, esophagus, pancreas, and rectum. The random sample was generated using an online random sequence generator (http://www.randomization.com). 


\section{Classification}

Two investigators (RR and HH), both board-certified surgeons, independently applied the definition and classification resulting from the Delphi process based on the information given in the operation records (protocol of surgery and of anesthesia). Additionally, postoperative complications were classified according to Dindo et al. [8] based on the entire patient record including the discharge letter. Disagreements were resolved by consensus.

\section{Practicability}

We evaluated practicability on a seven-point numeric scale.

\section{Data analysis}

Agreement between the judgment of the two raters (before resolving disagreements) was evaluated investigating raw categorical agreement (number of exact categorical matches between two raters divided by the total number of records) [17], kappa with quadratic weights [18, 19] and intraclass correlation [20].

\section{Results}

Delphi study

\section{Participants}

The link to the questionnaire was sent out to 52 experts, of whom 40 answered within the given deadline in both rounds (77\% return rate). In Delphi round 1, one participant submitted an incomplete questionnaire. Two additional contacted experts returned the questionnaire after the given deadline, and therefore their answers could not be included in the feedback to round 2 and the pilot evaluation, respectively (81\% return rate in total). Experts from 14 countries across the world from a variety of surgical disciplines and epidemiology and statistics took part in the study. Their median work experience was 25 years with an interquartile range (IQR) from 12 to 29 years (Table 1).

\section{Definition and classification}

The overall usefulness of a classification of intraoperative complications was rated as high (Fig. 1).

The Delphi study provided the following definition and classification of intraoperative complications (Table 2): any deviation from the ideal intraoperative course, given the indication for surgery and the interventions conform to current guidelines. The classification exclusively relates to any event occurring between skin incision and skin closure and should be rated directly after surgery. Any event during the index surgery must be considered, regardless whether it is related to surgery or anesthesia and regardless of preexisting risk factors, which should be captured along with the patients' baseline characteristics. The following events are not defined as intraoperative complications: (1) sequelae, i.e., effects inherent to current best practice surgery (such as the inability to walk after a leg amputation), (2) failures of cure (such as residual tumor after surgery), (3) events related to the underlying disease (such as bleeding from a ruptured abdominal aortic aneurysm or intraoperative sepsis due to a purulent peritonitis associated to a perforated appendicitis), (4) wrong-site or wrong-patient surgery or errors in indication (such as inappropriate indication for surgery according to current guidelines). (5) A deviation from the planned intervention due to unexpected intraoperative findings is not regarded as complication, if the initial indication for surgery and the deviation prompted by the findings conform to current guidelines. Details on the ratings by the experts regarding this distinction are given in Fig. 2. Complications evident only after skin closure are captured with the classification for postoperative complications.

The classification foresees four grades. Grade I applies to deviations from the ideal intraoperative course without the need for any additional treatment or intervention, e.g., a selflimiting bleeding at the trocar insertion site, an unintentional electrocautery injury to the parietal peritoneum, or a selflimiting arrhythmia. In grade II complications, an additional treatment or intervention is needed without the complication being life-threatening or leading to permanent disability. Examples are a redo-vascular anastomosis for bleeding, a suture of an iatrogenic bladder injury upon rectum surgery, a suture of a small bowel injury, or an antiarrhythmic treatment for cardiac arrhythmia. In the presence of life-threatening complications or permanent disability, the complication corresponds to grade III. Examples may be a hypovolemic shock due to laceration of the caval vein upon right adrenalectomy, an accidental trans-section of the femoral nerve, or an anaphylactic shock after routine antimicrobial prophylaxis. Events leading to death are classified grade IV, for instance a death from hypovolemic shock due to a large vessel injury or a death from myocardial infarction or central pulmonary embolism (Table 2). The classification is not intended to be dichotomized. Any report on intraoperative complications should state the number of raters involved in the assessment. In case of multiple raters, it should additionally be reported how a consensus was reached. It should further be stated whether the raters were involved in the procedure and if yes, in which role. Ratings by the experts concerning type of classification, timing of rating, resulting classification, and dichotomization are provided in Fig. 3. 
Table 1 Baseline

characteristics of participants in the Delphi study $(n=40)$

\begin{tabular}{|c|c|c|}
\hline Characteristic & Category & Value \\
\hline \multirow[t]{3}{*}{ Primary discipline, $n(\%)$} & Surgery & $37(92.5 \%)$ \\
\hline & Public health/epidemiology & $2(5.0 \%)$ \\
\hline & Statistics & $1(2.5 \%)$ \\
\hline \multirow[t]{16}{*}{ Primary subspecialty, $n(\%)$} & Breast surgery & $1(2.5 \%)$ \\
\hline & Cardiac surgery & $1(2.5 \%)$ \\
\hline & Colorectal surgery & $3(7.5 \%)$ \\
\hline & Neurosurgery & $1(2.5 \%)$ \\
\hline & Orthopedic surgery & $4(10 \%)$ \\
\hline & Pediatric surgery & $2(5.0 \%)$ \\
\hline & Plastic and reconstructive surgery & $1(2.5 \%)$ \\
\hline & Surgical oncology & $1(2.5 \%)$ \\
\hline & Thoracic surgery & $2(5.0 \%)$ \\
\hline & Traumatology & $1(2.5 \%)$ \\
\hline & Urology & $1(2.5 \%)$ \\
\hline & Vascular surgery & $2(5.0 \%)$ \\
\hline & Visceral surgery & $14(35 \%)$ \\
\hline & Other surgical specialty & $3(7.5 \%)$ \\
\hline & Epidemiology & $2(5.0 \%)$ \\
\hline & Statistics & $1(2.5 \%)$ \\
\hline \multirow[t]{14}{*}{ Country, $n(\%)$} & Switzerland & $23(57.5 \%)$ \\
\hline & Germany & $1(2.5 \%)$ \\
\hline & Austria & $2(5.0 \%)$ \\
\hline & Netherlands & $1(2.5 \%)$ \\
\hline & Ireland & $1(2.5 \%)$ \\
\hline & United Kingdom & $3(7.5 \%)$ \\
\hline & France & $2(5.0 \%)$ \\
\hline & Italy & $1(2.5 \%)$ \\
\hline & Spain & $1(2.5 \%)$ \\
\hline & Turkey & $1(2.5 \%)$ \\
\hline & USA & $1(2.5 \%)$ \\
\hline & Singapore & $1(2.5 \%)$ \\
\hline & Hongkong & $1(2.5 \%)$ \\
\hline & Australia & $1(2.5 \%)$ \\
\hline \multirow{3}{*}{$\begin{array}{l}\text { Involved in clinical research as } \\
\quad \text { (several options applicable), } n(\%)\end{array}$} & Principal investigator & $37(92.5 \%)$ \\
\hline & Co-investigator & $35(87.5 \%)$ \\
\hline & Biostatistician & $2(5.0 \%)$ \\
\hline \multicolumn{2}{|c|}{ Years of overall work experience, median (IQR) } & $25(12-29)$ \\
\hline \multicolumn{2}{|c|}{ Years of surgical work experience, median $(\mathrm{IQR})^{\mathrm{a}}$} & $24(12-29)$ \\
\hline \multicolumn{2}{|c|}{ Years of clinical research experience, median (IQR) } & $20(8-24)$ \\
\hline \multirow[t]{2}{*}{ Gender, $n(\%)$} & Male & $36(90 \%)$ \\
\hline & Female & $4(10 \%)$ \\
\hline
\end{tabular}

Assessed in round 1

$I Q R$ interquartile range

${ }^{\text {a }} n=3$ with primary nonsurgical discipline excluded male, 23 (38\%) had an ASA (American Society of Anesthesiologists) score of three or higher, and 17 (28\%) were emergency admissions. Type A surgery was represented by inguinal/femoral hernia repair $(n=10)$ and thyroid/parathyroid surgery $(n=10)$, type B surgery by cholecystectomy $(n=10)$ and colon surgery $(n=10)$, and 


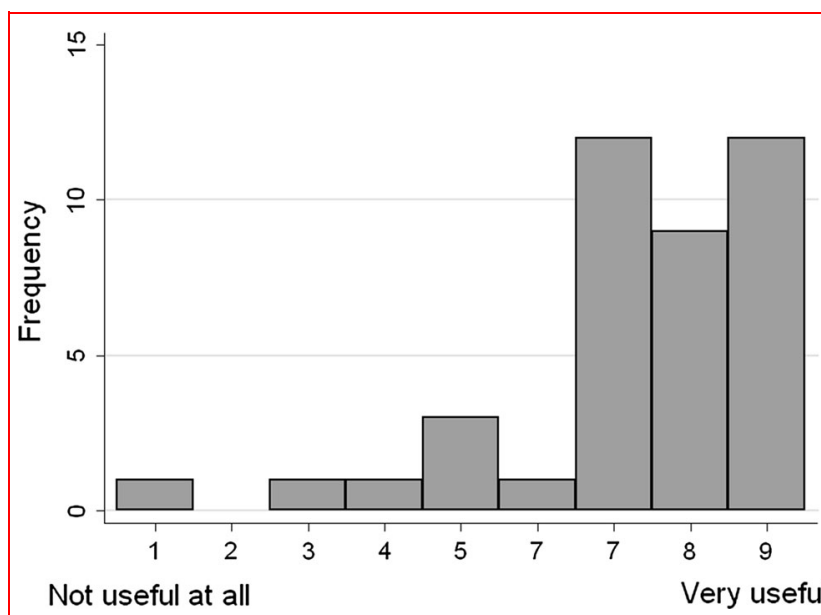

Fig. 1 Delphi study round 1: answers to the question concerning overall usefulness of a classification of intraoperative complications: how do you rate the overall usefulness of a classification of intraoperative complications ( 1 not useful at all, 9 very useful)?

type C surgery by major liver $(n=5)$, pancreatic $(n=5)$ esophageal $(n=5)$, and rectal surgery $(n=5)$ (online resource 1).

\section{Outcomes}

The median length of hospital stay was 8 days (IQR 3-15). Based on the judgment after resolving disagreement, intraoperative complications were recorded in $19(32 \%)$ and postoperative complications in $27(45 \%)$ patients. The spread of complications over the grades was higher for postoperative than for intraoperative complications due to the higher number of low grade intraoperative complications. Details of the grade and outcomes by complexity of the intervention are given in online resource 1 .

\section{Agreement}

The raw categorical agreement between the two investigators was $87 \%$ for both intra- and postoperative complications. Details of the kappa statistics and intraclass correlation coefficient are provided in Table 3.

\section{Practicability}

Both investigators rated the practicability of the definition as well as of the classification as 6 on the scale between one and seven.

\section{Discussion}

The Delphi study provides a comprehensive definition of intraoperative complications as any deviation from the ideal intraoperative course occurring between skin incision and skin closure, given that the indication for surgery and the interventions conform to current guidelines. The classification should be applied directly after surgery. The pilot study demonstrated practicability as well as a good interrater agreement.

Table 2 Proposed Classification of Intraoperative Complications (CLASSIC)

\begin{tabular}{ll}
\hline Grade & Definition \\
\hline & $\begin{array}{l}\text { The classification exclusively relates to any event occurring between skin incision and skin closure and should } \\
\text { be rated directly after surgery. Any event during the index-surgery must be considered, regardless } \\
\text { whether it is surgery or anesthesia-related }\end{array}$ \\
& $\begin{array}{l}\text { Prerequisite: the indication for surgery and the interventions conform to current guidelines } \\
\text { No deviation from the ideal intraoperative course }\end{array}$ \\
Grade 0 & Any deviation from the ideal intraoperative course \\
Grade I & - Without the need for any additional treatment or intervention \\
Grade II & Any deviation from the ideal intraoperative course \\
& - With the need for any additional treatment or intervention \\
Grade III & Not life-threatening and not leading to permanent disability \\
& Any deviation from the ideal intraoperative course \\
- With the need for any additional treatment or intervention & - Life-threatening and/or leading to permanent disability \\
Grade IV & Any deviation from the ideal intraoperative course \\
& - With death of the patient
\end{tabular}

a The following events are not defined as intraoperative complications: sequelae, failures of cure, events related to the underlying disease, wrong-site or wrong-patient surgery, or errors in indication 


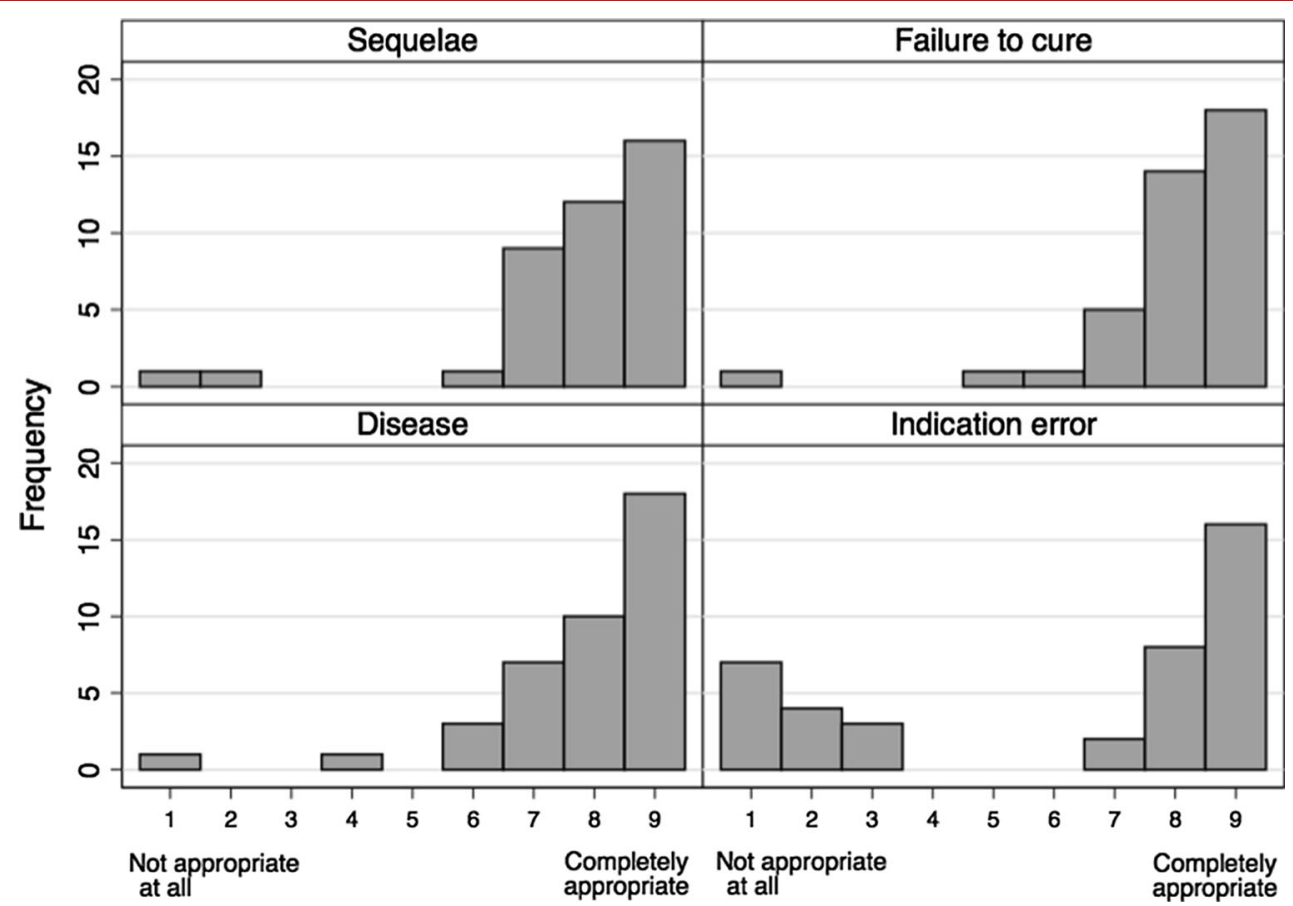

Fig. 2 Delphi study round 2: answers to the questions concerning the distinction of intraoperative complications from sequelae, failure to cure, events related to the underlying disease, and error in indication: based on these answers of round 1, we kindly ask you to rate the following updated definition ( 1 not at all appropriate, 9 completely appropriate): Sequelae: a definition of intraoperative complications needs to distinguish complications from sequelae, i.e., effects inherent to current best practice surgery (such as the inability to walk after amputation of a leg). It should refer to the index surgery and not to potential follow-up surgeries to treat a complication. Failure to cure: a definition of intraoperative complications needs to distinguish complications from failures of cure (such as residual

Relation to other studies developing classification systems

Many previous attempts to address intraoperative complications origin in either human factor and system errors perspectives or in individual surgeon performance related to surgical education, surgical experience, and technical skills. This is reflected by a review of studies describing technical errors during laparoscopy, in which error analysis was applied in $38 \%$ and surgical performance quantified in an educational setting in $62 \%$ [21].

Fabri et al. developed a classification system of errors in operative therapy in an iterative process, interacting with faculty members of one major surgical university department [22]. The system was then validated threefold: by the personal judgement of practicability of four experts $(50 \%$ return rate), by evaluation of agreement between two senior academic surgeons scoring 72 incidences, and by a national survey rating three scenarios. There, medical errors were evaluated according to the time-point of occurrence and the tumor after surgery), under the condition the indication for surgery and the interventions conform to current guidelines. Disease: a definition of intraoperative complications needs to distinguish complications from events related to the underlying disease (such as bleeding from a ruptured abdominal aortic aneurysm or intraoperative sepsis due to a purulent peritonitis associated to a perforated appendicitis), under the condition the indication for surgery and the interventions conform to current guidelines. Indication error: a definition of intraoperative complications should NOT refer to wrong-site or wrong-patient surgery and NOT to errors in indication (such as inappropriate indication for surgery according to current guidelines)

type of errors as well as how likely the patient outcome was attributable to the error. Additionally, the adverse outcome was graded depending on the degree of injury and the prolongation of length of hospital stay. Agreement was found to be over $70 \%$ in all error categories in both the paired comparison and the national survey (return rate $29.5 \%$ ). The system was then applied to over 9,000 surgical procedures. The results suggested that complications are mainly caused by individual surgeons. The study was however controversially discussed, since some of the complications caused by individual surgeons could still have their origin in latent factors in the system [23]. Our study shows similar results concerning interrater agreement. The definition and classification are however not strictly comparable, since our system is focussing on the time between skin incision and closure, relying on a second system to capture postoperative events.

In comparison, the classification for postoperative complications proposed by Dindo et al. [8] was validated as follows: first, in a sample of over 6,000 patients, the 


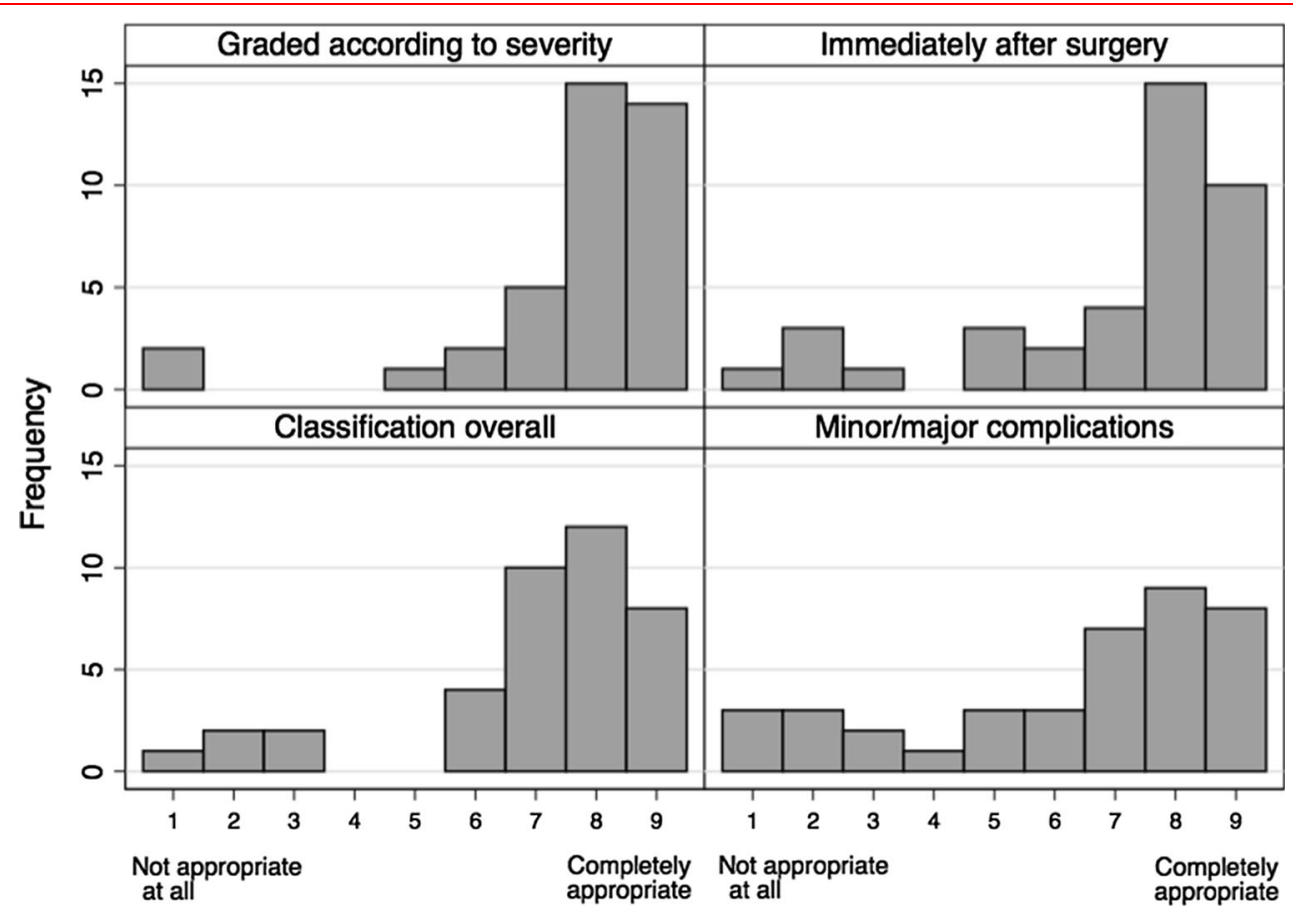

Fig. 3 Delphi study round 2: answers to the questions concerning type of grading, classification overall, time-point of grading, dichotomisation. We kindly ask you to rate the following ( 1 not at all appropriate, 9 completely appropriate): Graded according to severity: a classification of intraoperative complications should consider the severity of the complication and take into account both the necessary treatment and permanent sequelae resulting from the complication. Immediately after surgery: should it be possible to definitively fill in a classification of intraoperative complications directly after surgery, whereas complications apparent only after surgery are handled with a classification system for postoperative complications? Classification overall: we kindly ask you to rate the updated classification: prerequisite for all grades: under the condition the indication for surgery and the interventions conform to current guidelines. Grade 0: no preventable deviation (an unpreventable deviation could be for instance a deviation due to an unexpected anatomical situation); grade 1: preventable deviation from the ideal intraoperative course without the need for any additional treatment or intervention; grade 2 and 3 with the need for any additional treatment or intervention, grade 3 if life-threatening and/or leading to permanent disability, else grade 2; grade 4 with patient death. Minor/major complications: should complications needed to be dichotomised for further analysis (which we not necessarily advocate), we propose to summarize grades I-II together as "minor complications" versus grades III-IV as "major complications"

Table 3 Agreement of the pilot study ( $n=60$ cases, $n=2$ raters)

\begin{tabular}{llll}
\hline Characteristic & Category & Intraoperative complications $^{\mathrm{a}, \mathrm{b}}$ & Postoperative complications $^{\mathrm{c}}$ [3] \\
\hline Kappa statistics & Agreement (\%) & 87 & 87 \\
& Kappa (95 \% CI) & $0.72(0.48-0.89)$ & $0.79(0.69-0.94)$ \\
& Quadratic weighted kappa (95 \% CI) & $0.83(0.73-0.94)$ & $0.97(0.94-1.00)$ \\
Intraclass correlation & Intraclass correlation $(95 \% \mathrm{CI})$ & $0.83(0.73-0.90)$ & $0.97(0.95-0.98)$ \\
\hline
\end{tabular}

CI confidence interval

${ }^{\text {a }}$ Based on agreement of both reviewers

b Classification applied immediately postoperatively without including the option "potentially" life-threatening/leading to permanent disability

c Based on agreement of both reviewers

correlation between the severity grades of the classification and the complexity of surgery as well as the length of hospital stay was evaluated and found to be high; second, acceptability and reproducibility based on 14 clinical cases were assessed in an international survey. Acceptability was high, and the answers to the scenarios were correct in $90 \%$, independent of the level of training or origin of the surgeon. 
Strengths and limitations

To the best of our knowledge, there is no validated definition and classification specifically addressing intraoperative complications, while there are several classification systems for postoperative complications. With the involvement of a diverse team of international experts and a high return rate, we were able to launch an extensive discussion about the definition and classification of intraoperative complications. It became evident that the ideal choices were not uniformly the most practical; hence, some decisions were rather pragmatic, resulting in a comprehensive definition and rather simple classification. We are well aware that we are presenting results of the Delphi process and pilot study, which may be subject to further refinement upon full-scale validation. Moreover, the retrospective setting of the pilot study with its small sample is unlikely to cover all possible complications. This is supported by the findings of a review of over eight-thousand interventions, where operative reports missed $13 \%$ and discharge summaries missed $14 \%$ of complications [24]. Moreover, we found a lower spread of complications across grades for intra- than for postoperative complications, thus our similar interrater agreement for intra- and postoperative complications needs to be interpreted with care. As further research, we intend to conduct a full-scale validation of this instrument in a prospective international multicenter cohort.

\section{Implications for current practice and further research}

In order to enhance patient safety and transparency in reporting, there is an urgent need for standardized reporting of intraoperative events.

In an evaluation of surgical adverse event reporting with a specific focus on surgical site infection, anastomotic leak, deep vein thrombosis, and surgical mortality, important differences in definitions and duration of follow-up were found, hampering comparisons [25]. In a previous study of our group based on a cohort of 46 randomized controlled trials published in surgical journals, only half of the studies provided exact definitions of postoperative complications, and only $13 \%$ of the studies provided definitions of intraoperative complications [12].

Standardization of the definition and classification of intraoperative complications are important in a number of settings: first, in research and development, when assessing new surgical techniques and devices; second, in educational settings (individual performance) and institution benchmarking; third, for risk management, capturing intraoperative events with an increased risk of postoperative complications within a critical incidents reporting system thus enabling to take actions to address such incidents $[1,2$, 5] or in clinical research where postoperative event rates may be too low to capture potential safety concerns; and last for patient management, enhancing quality of patient handover after surgery with anticipated postoperative problems [3, 4].

Although several attempts have been undertaken to find new reimbursement strategies in the light of increasing health care cost, based on pay-for-performance initiatives and risk-sharing between payer and provider [26, 27], translating these attempts to surgical interventions might be quite challenging. There, not only the intraoperative phase would then need to be considered, but also the postoperative phase as well as patient risk factors. We thus believe that this is currently beyond the scope of such a classification. As a matter of fact, it is crucial to interpret any reports using such a classification system in the light of patient risk factors, as captured with their baseline characteristics (e.g., intraoperative arrhythmia in a patient with coronary artery disease or bleeding in a patient with anticoagulation therapy).

We believe that this classification can be used prospectively and retrospectively, i.e., with direct rating after surgery, based on the operation record or on videorecordings. When used retrospectively, the limitation of not capturing all complications due to the likely absence of low grade complications has to be kept in mind. We expect variability in reporting of grade I complications. In these instances, grade 0 and I complications could be taken together for subsequent analysis. In some research or educational settings (e.g., learnings from low grade complications that may be transferable to more critical situations), however, grade I complications may be of interest as stand-alone category. As further research, we plan to refine and validate the proposed definition and classification in a multinational, prospective cohort study. We will evaluate whether there is an association between the classification and the complexity and duration of surgery. Furthermore, an explorative analysis shall evaluate whether the classification is predictive for a number of adverse postoperative outcomes, adjusted for the most relevant confounders. Last, we are aware of the fact that as a result of the Delphi process, our classification includes only four grades with death corresponding to grade 4. In contrast, classifications for postoperative complications such as the widely adopted Clavien-Dindo classification [7, 8] include five grades with death corresponding to grade 5 . This might lead to confusion, so we intend to re-address this issue within the validation study. 


\section{Conclusions}

We provide a comprehensive definition of intraoperative complications and a straightforward classification with a high interrater agreement and practicability, as evaluated in a pilot study. This classification shall now undergo fullscale validation.

With the proposed work we are contributing to standardized reporting in clinical practice, research, and educational settings, ultimately enhancing patient safety and allowing for informed healthcare decision making.

Acknowledgments We are very grateful to all experts who participated in this study. Their input was very important in the development of the proposed definition and classification.

Conflict of interest No financial support was received for this study. Heiner C. Bucher is supported by Grants from Santésuisse and the Gottfried and Julia-Rhyner-Foundation. There are no conflicts of interest to declare. Rachel Rosenthal is an employee of F. HoffmannLa Roche Ltd. since May 01, 2014. The present study was conducted before Rachel Rosenthal joined F. Hoffmann-La Roche Ltd. and has no connection to her employment by the company.

\section{References}

1. Kram R (2008) Critical incident reporting system in emergency medicine. Curr Opin Anaesthesiol 21:240-244

2. Leape LL (1994) Error in medicine. JAMA 272:1851-1857

3. Manser T, Foster S, Flin R, Patey R (2013) Team communication during patient handover from the operating room: more than facts and figures. Hum Factors 55:138-156

4. Nagpal K, Abboudi M, Fischler L, Schmidt T, Vats A, Manchanda C, Sevdalis N, Scheidegger D, Vincent C, Moorthy K (2011) Evaluation of postoperative handover using a tool to assess information transfer and teamwork. Ann Surg 253:831-837

5. Reason J (2000) Human error: models and management. BMJ 320:768-770

6. Clavien PA, Sanabria JR, Strasberg SM (1992) Proposed classification of complications of surgery with examples of utility in cholecystectomy. Surgery 111:518-526

7. Clavien PA, Barkun J, de Oliveira ML, Vauthey JN, Dindo D, Schulick RD, de SE, Pekolj J, Slankamenac K, Bassi C, Graf R, Vonlanthen R, Padbury R, Cameron JL, Makuuchi M (2009) The Clavien-Dindo classification of surgical complications: five-year experience. Ann Surg 250:187-196

8. Dindo D, Demartines N, Clavien PA (2004) Classification of surgical complications: a new proposal with evaluation in a cohort of 6336 patients and results of a survey. Ann Surg 240:205-213
9. Pillai SB, van Rij AM, Williams S, Thomson IA, Putterill MJ, Greig S (1999) Complexity- and risk-adjusted model for measuring surgical outcome. Br J Surg 86:1567-1572

10. Pomposelli JJ, Gupta SK, Zacharoulis DC, Landa R, Miller A, Nanda R (1997) Surgical complication outcome (SCOUT) score: a new method to evaluate quality of care in vascular surgery. J Vasc Surg 25:1007-1014

11. Strasberg SM, Linehan DC, Hawkins WG (2009) The accordion severity grading system of surgical complications. Ann Surg 250:177-186

12. Rosenthal R, Hoffmann H, Dwan K, Clavien PA, Bucher HC (2015) Reporting of adverse events in surgical trials: critical appraisal of current practice. World J Surg 39:80-87

13. Hsu C-C, Sandford BA (2013) The Delphi technique: making sense of consensus. Pract Assess Res Eval 12(10):1-8

14. Preston CC, Colman AM (2000) Optimal number of response categories in rating scales: reliability, validity, discriminating power, and respondent preferences. Acta Psychol 104:1-15

15. Miller GA (1956) The magical number seven plus or minus two: some limits on our capacity for processing information. Psychol Rev 63:81-97

16. Klotz HP, Candinas D, Platz A, Horvath A, Dindo D, Schlumpf R, Largiader F (1996) Preoperative risk assessment in elective general surgery. Br J Surg 83:1788-1791

17. Streiner DL (1995) Learning how to differ: agreement and reliability statistics in psychiatry. Can J Psychiatry 40:60-66

18. Fleiss JL, Cohen J (1973) Equivalence of weighted kappa and intraclass correlation coefficient as measures of reliability. Educ Psychol Measur 33:613-619

19. Warrens MJ (2011) Weighted kappa is higher than Cohen's kappa for tridiagonal agreement tables. Stat Methodol 8:268-272

20. Shrout PE, Fleiss JL (1979) Intraclass correlations: uses in assessing rater reliability. Psychol Bull 86:420-428

21. Bonrath EM, Dedy NJ, Zevin B, Grantcharov TP (2013) Defining technical errors in laparoscopic surgery: a systematic review. Surg Endosc 27:2678-2691

22. Fabri PJ, Zayas-Castro JL (2008) Human error, not communication and systems, underlies surgical complications. Surgery 144:557-563

23. Nagpal K, Vats A, Vincent C, Moorthy K (2009) A systems approach to errors. Surgery 145:689-690

24. Platz J, Hyman N (2012) Tracking intraoperative complications. J Am Coll Surg 215:519-523

25. Bruce J, Russell EM, Mollison J, Krukowski ZH (2001) The measurement and monitoring of surgical adverse events. Health Technol Assess 5:1-194

26. Edlin R, Hall P, Wallner K, McCabe C (2014) Sharing risk between payer and provider by leasing health technologies: an affordable and effective reimbursement strategy for innovative technologies? Value Health 17:438-444

27. Long G, Mortimer R, Sanzenbacher G (2014) Evolving provider payment models and patient access to innovative medical technology. J Med Econ 17:1-11 